\title{
Planetary Nebulae, globular Clusters \\ AND THE GALACTIC HALO
}

\author{
SIDNEY VAN DEN BERGH \\ David Dunlap Observatory, University of Toronto, Richmond Hill, Ontario, Canada
}

\begin{abstract}
Planetary nebulae are used as a tracer for halo Population II stars. A comparison of the number of planetary nebulae in the galactic pole caps $\left(|b| \geqslant 45^{\circ}\right)$ with the number of planetaries in globular clusters suggests that (to within a factor of 2 or 3) the galactic halo has a luminosity $L_{B} \simeq 2 \times 10^{8} L_{\odot}$. From the work of Oort it is estimated that the galactic halo has a mass of at least $6 \times 10^{9} \mathfrak{M} \odot_{\odot}$ so that $\mathfrak{M} / L>30$ for the galactic halo.
\end{abstract}

Planetary nebulae are generally regarded as members of the old disk population (O'Connell, 1958) of the Galaxy. Nevertheless there are some planetary nebulae which are undoubtedly members of a metal-poor halo population. The best known examples of this type of object are the planetary nebulae K 648 in M15 (Küstner, 1921; Pease, 1928; O'Dell et al., 1964; Peimbert, 1973) and the high-velocity halo planetary $49+88^{\circ} 1$, which has been shown to be metal-poor by Miller (1969).

It is the purpose of this paper to point out that high-latitude planetary nebulae, for which observational data should be quite complete, can be used as a tracer for halo Population II. For the purpose of the present investigation we shall define halo Population II as that population component of the Galaxy which has a space distribution similar to that of the cloud of globular clusters in which the Galaxy is embedded.

According to Arp (1965) the Galaxy contains 119 globular clusters of which 16 (13 percent) are located in the northern $\left(b \geqslant+45^{\circ}\right)$ and southern $\left(b \leqslant-45^{\circ}\right)$ pole caps. A total of 8 planetary nebulae (Perek and Kohutek, 1967) are known within this same area; four in the north pole cap and four in the south pole cap. This sample of planetary nebulae at high latitudes should be virtually complete because this area has been thoroughly searched with powerful instruments such as the Palomar 48-in. Schmidt (Abell, 1966).

Of the 8 known planetary nebulae with $|b| \geqslant 45^{\circ}$ at least three (NGC 246, NGC 1360 and NGC 3587) are relatively nearby objects within $600 \mathrm{pc}$ of the Sun (Cahn and Kaler, 1971), which are probably members of the galactic disk population. The total number of true halo-type high-latitude $\left(|b| \geqslant 45^{\circ}\right)$ planetaries is therefore $\simeq 5$, Assuming such objects to have a space distribution similar to that of globular clusters then yields a total population of $5 \times(119 / 16)=37$ such objects in the entire Galaxy.

So far K 648 in M15 is the only planetary that is known to be located in a globular cluster. A search for additional planetaries in globular clusters by Feibelman (unpublished) and by Andrews (Thackeray, 1971) has so far remained fruitless. In what follows it will, more or less arbitrarily, be assumed that the total number of planetary nebulae in all galactic globular clusters is three. The total population of bright evolved stars in the halo is therefore very approximately $(37 / 3) \simeq 12$ times greater than 
that in all galactic globular clusters together. The total (blue) luminosity of all galactic globular clusters is $\simeq 1.7 \times 10^{7} L_{\odot}$ so that the total luminosity of the halo component of the Galaxy becomes $\simeq 2 \times 10^{8} L_{\odot}$. (This value is quite insensitive to the luminosity function of faint $\mathrm{K}$ and $\mathrm{M}$ dwarfs which might differ in globular clusters and in the halo).

Oort (1965) has presented powerful arguments which suggest that at least 5 percent of the mass of the Galaxy is contributed by faint halo subdwarfs of spectral types $\mathrm{K}$ and M. Adopting a total galaxy mass of $1.3 \times 10^{11} \mathfrak{M}_{\odot}$ (Innanen, 1966) then yields a mass $\geqslant 6.5 \times 10^{9} \mathfrak{M}_{\odot}$ for the halo. Comparison of this value with the halo luminosity obtained above yields $\mathfrak{M} / L>30$ for the galactic halo. This is very much larger that the value $\mathfrak{M} / L \simeq 0.5$ that is obtained for galactic globular clusters (Schwarzschild and Bernstein, 1955; Feast and Thackeray, 1960). The fact that $\mathfrak{M} / L>30$ for the halo and $\mathfrak{M} / L \simeq 0.5$ for globular clusters militates against the hypothesis (Peebles and Dicke, 1968; Peebles, 1969) that the halo was formed from gas clouds in which physical conditions were similar to those prevailing in proto-globular clusters.

The high $\mathfrak{M} / L$ ratio that is found in the galactic halo might be related to the high $\mathfrak{M} / L$ ratios that have been observed in the outer regions of M31 and M33. In M33 Boulesteix and Monnet (1970) find that the mass-to-light ratio changes from $\mathfrak{M} / L \simeq 2.5$ at $\varpi=5^{\prime}$ to $\mathfrak{M} / L \simeq 20$ at $\varpi=40^{\prime}$. A similar trend in the mass-to-light ratios in M31 has been found by Gottesman and Davies, (1970).

According to van den Bergh (1972) the Galaxy has a total luminosity $L_{B}=(1.1 \pm$ $\pm 0.3) \times 10^{10} L_{\odot}$. Comparison with the halo luminosity $L_{B} \simeq 2 \times 10^{8} L_{\odot}$ that was obtained above indicates that $\sim 2$ percent of the luminosity of the Galaxy is contributed by metal-poor halo stars.

\section{References}

Abell, G. O.: 1966, Astrophys. J. 144, 259.

Arp, H. C.: 1965, Stars and Stellar Systems 5, 401

Bergh, S. van den: 1972, Astron. Astrophys. (in press).

Boulesteix, J. and Monnet, G.: 1970, Astron. Astrophys. 9, 350.

Cahn, J. H. and Kaler J. B.: 1971, Astrophys. J. Suppl. 22, 319, (No. 189).

Feast, M. W. and Thackeray, A. D.: 1960, Monthly Notices Roy. Astron. Soc. 120, 463.

Gottesman, S. T. and Davies, R. D.: 1970, Monthly Notices Roy. Astron. Soc. 149, 263.

Innanen, K. A.: 1966, Z. Astrophys. 64, 158.

Küstner, F.: 1921, Veröff. astro. Inst. Univ. Bonn 15, 1.

Miller, J. S.: 1969, Astrophys. J.157, 1215.

O'Connell, D. J. K.: 1958, Stellar Populations, North Holland Pub. Co., Amsterdam, p. 533.

O'Dell, C. R., Peimbert, M., and Kinman, T. D.: 1964, Astrophys. J. 140, 119.

Oort, J. H.: 1965, IAU Trans. XIIA, 789.

Pease, F. G.: 1928, Publ. Astron. Soc. Pacific 40, 342.

Peebles, P. J. E.: 1969, Astrophys. J. 157, 1075.

Peebles, P. J. E. and Dickie, R. H.: 1968, Astrophys. J. 154, 891

Peimbert, M.: Paper presented at the 18th Liège Symposium June 26-28, 1972.

Perek, L. and Kohoutek, L.: 1967, Catalogue of Galactic Planetary Nebulae, Academia Publ. House, Prague.

Schwarzschild, M. and Bernstein S.: 1955, Astrophys. J. 122, 200.

Thackeray, A. D.: 1971, Quart. J. Roy. Astron. Soc. 12, 320. 


\section{DISCUSSION}

Feast: (1) Griffin's radial velocities will possibly increase $\mathfrak{M} / L$ for globular clusters by a moderately large factor. (2) It is important in your calculations that the ratio of planetaries in the region of galactic centre to globular clusters there is much greater than in the halo.

van den Bergh: The observations by Wilson and Coffeen (Astrophys. J.119, 197, 1954) and by Griffin (Observatory 92, 29, 1972) refer to different clusters. For M92 Wilson and Coffeen obtain a radial velocity dispersion of $4.4 . \mathrm{km} \mathrm{s}^{-1}$ compared to Griffin's value of $6 \mathrm{~km} \mathrm{~s}^{-1}$ in M13. This small difference in velocity dispersion may, at least in part, be due to the fact that M13 is a more compact cluster (Arp, Stars and Stellar Systems 5, 401, 1965) than is M92. In summary there is as yet no indication that the published $\mathfrak{M} / L$ ratios in globular clusters need to be revised significantly.

(2) The nuclear bulge of the Galaxy (van den Bergh, Publ. Astron. Soc. Pacific 84, 306, 1972) contains a dominant old disk population in which a few halo population stars are embedded. The vast majority of the planetary nebulae in the direction of the galactic centre should therefore belong to the old disk population.

Demarque: Would Dr Schwarzschild care to comment on a recent study done at Princeton by Ostriker and Peebles which suggests that the galactic halo is much more massive than previously believed? [No reply.]. 